\title{
COVID-19 and Mental Stress among the Urban Youths of Dhaka City
}

\begin{abstract}
The COVID-19 pandemic has imposed threats on both physical and mental health since its outbreak. This paper aims to highlight the mental and physical health challenges amongst the home-quarantined youths of Dhaka city. A total of 150 young adults were surveyed, within the age range of (12-30). The participation was voluntary and was completed online.

The findings suggest, many youngsters are currently suffering a wide range of mental health issues related to anxiety, fear, isolation, depression, feeling lonely, emotional outburst, insomnia, and other sleeping/ eating habit disturbances during COVID 19 pandemic. Many are emotionally vulnerable after losing their close ones, losing jobs, uncertain education or by staying indoors for months. Many get agitated wearing protective gear like masks or using sanitizers.

The panic caused by the infection from physical proximity has reached such a level that everything now is shifted from physically going out to virtual conferences and many other significant shifts in people's behavior. But, in spite of all the trauma and negative affects the pandemic has put us through, there is some hope that the new focus on mental health issues may reduce stigma and increase availability of support services.

Finally the paper recommends some way forward for effective mitigation against the harmful effects of limited or indoor physical activity on mental and physical health due to social distancing and quarantine based on the collected data.
\end{abstract}


Key words: Coronavirus, Covid-19, Youth, Young, Mental health, Psychological health, Lockdown

\section{Introduction}

Transmittable disease outbreaks, like the current Coronavirus disease 2019 (COVID-19), caused by a novel coronavirus, SARS-CoV-2 (severe acute respiratory syndrome coronavirus-2) has plunged the world into chaos and induced an all-pervasive sense of panic. As the pandemic speedily spreads across the world, it is inducing considerable mental and health risks. Creating fear, anxiety, and concern in the population at large and among certain groups in particular, such as young outgoing people, older adults, care providers and people with underlying health conditions. The lockdown is affecting all groups of people in the world either physically or mentally. However, developing countries with a poorly structured health sector and a high population density are struggling to cope with the challenges.

Especially being indoors for months are hampering many people's usual routines. According to a report from the National Academies of Sciences: "Social isolation has been associated with a significantly increased risk of premature mortality from all causes," including a " $50 \%$ increased risk of developing dementia;" a " $29 \%$ increased risk of incident coronary heart disease;" a " $25 \%$ increased risk for cancer mortality;" a " $59 \%$ increased risk of functional decline;" and a " $32 \%$ increased risk of stroke." (Huemer et al., 2009).

And an increased level of loneliness, depression, anxiety, eating disorder, sleeping disorder, consumption of harmful alcohol as well as other risk behaviors including those that relate to sexual behavior, substance abuse, self-harm, and turning violent or angry frequently are seen.

The study aims to further our understanding of how the nationwide lockdown ordered to combat Covid-19 is affecting the youth of Dhaka city psychologically. As well as reviewing the harmful effects of limited outdoor or physical activity due to social distancing and quarantine based on the findings. 


\section{Problem Statement}

About 47.6 million i.e. $30 \%$ of the total 158.5 million people in Bangladesh are young and considered to be part of the youth. Poor mental health can have effects on wider health and development at a young age and can be associated with several health and social outcomes such as higher alcohol, tobacco and illicit substances use, adolescent pregnancy, education dropout, involvement in crime, rude behaviors, etc. There is a huge consensus that healthy development contributes to good mental health and can prevent mental health problems. Enhancing social skills, problem-solving skills and self-confidence can help prevent mental health problems like anxiety and depression. Since the lockdown, many have faced challenges. Seeing the virus spread so drastically has shaken countless lives from within.

To avoid the further spread of the COVID-19 pandemic, everyone is bound to stay at home or follow all the safety precautions while outside. And this situation is not very desirable, especially for the young generation. Thus, young people both who have been affected by the virus and the ones who are still safe face problems like feeling anxious, annoyed, get angry easily, insomnia, sleeping disorder, eating disorder, always in fear that someone close might be affected with the virus, many are depressed not being able to socialize and facing a financial crisis as necessity goods have become scarce and expensive with rising demand. Staying indoors has further brought a change in everyone's lifestyle.

Which along with mental stress and anxiety is seeding physical sickness like obesity, dementia, diabetes, etc. Proper care and a healthy lifestyle are needed to restore the mental health and fight back against viruses.

\section{Summary of the Literature}

The COVID-19 pandemic and the resulting economic recession have negatively affected many people's mental and physical health creating new barriers for people already suffering from illness 
or isolation. Thinking about the severity of this situation, many doctors, practitioners and researchers are analyzing the impacts and possible outcomes of this serious issue. There is currently not much literature addressing the health impacts of COVID-19 on the general young population of Dhaka city. A global, novel virus that keeps us contained in our homes for months is more threatening than it seems. Five papers have directly addressed how this global epidemic has brought a shift in our health conditions.

A paper published in May 2020 [4], illustrates that a higher level of stress amongst the young and educated is seen. The study was carried out by collecting data via an online survey of 1427 Bangladeshis. Similarly, another paper published on July 2020 [8], based on previous literature show that more than one-third of the people in China had generalized anxiety disorders, $18 \%$ of the people suffered from a depressive illness and poor sleep quality. Majority of them were young, being less than 35 years old. This study further shows that lockdown in Bangladesh has caused job insecurity which is provoking disturbance in the mental health of many.

Findings from another article of April 2020[11], prepared via an online survey on the general population of Bangladesh gives out the interventions of Covid-19 recommended by Inter-Agency Standing Committee. Besides, another publication from August 2020 [12]. From the data collected via a health tracking poll in the United States of America shows, 62\% of adults with fair or poor health status reported negative mental health impacts compared to $51 \%$ of adults with excellent, very good, or good health status.

And lastly, a paper prepared from a survey conducted on 5070 adult participants of Australia in July 2020 [10]. The findings showed, $26 \%$ of people surveyed dealt with a prior history of mental health issues, and $11 \%$ of those without pre-existing mental health issues reported elevated health anxiety in the, which is higher than rates of health anxiety in the general Australian population (3.4\%), and closer to the rates of health anxiety observed in general practice (10\%) and outpatient medical clinic settings (20-25\%). 


\section{Consequences of Covid-19 in Bangladesh Context}

The COVID-19 pandemic has been declared an International public health emergency by the World Health Organization. Similarly, the Government of Bangladesh imposed a closure of all educational institutions and both public and private offices, and a nationwide lockdown from $26^{\text {th }}$ March, 2020 in an effort to control the spread of the outbreak. Public gatherings were also banned (WHO 2020c), and travel from countries with high transmission risk, such as China, Iran, and Italy, was suspended (Anadolu 2020). Despite these efforts, COVID-19 has reached all 64 administrative districts in Bangladesh by July 1st 2020, causing over 145,000 cases and 1,874 deaths. (IEDCR 2020). High population density, deficient personal hygiene practices, and poor economic conditions make the majority of the Bangladeshi population particularly vulnerable to this virus.

The impact of social distancing and isolation on physical and mental health has been acute worldwide. The effect is much more severe for the middle-class and lower-income people of developing countries like Bangladesh.

\section{Research Methodology}

This study aimed to obtain a random sample group of one hundred fifty (150) young adults aged (12-30). Participants have been obtained from Dhaka city. The sampling method was snowballing or chain referral sampling. The data was estimated using a self-reporting questionnaire. The literature review was conducted with published e-journals, downloaded from Google, Google Scholar and Pub med. 


\section{Materials for the survey:}

A prospective cross-sectional web-based survey created on Google forms was conducted to assess the psychological response of 150 young adults of Dhaka city belonging from different backgrounds, livelihoods, and occupation via social media platforms from May 15th to August 15th 2020. As there was no scope of going out because of the nationwide lockdown. All the surveys were included in analyses to obtain more precise results. As age was an exclusion criterion, all the survey takers were between 12- 30 years.

An anonymous online questionnaire (Appendix I) was developed after the literature study on Covid-19, mental and physical health papers. All the papers and publications were downloaded from authentic sites. As the survey questionnaire was in English, all the questions were straight forward and in easy English. And the Survey respondents were notified to contact the author if they faced any difficulties while answering or understanding the survey tool.

The survey included a short outline of the study context, purpose, confidentiality agreement, and informed consent. Clicking on the survey link directed the participants first to the study overview and informed consent. Demographic information was required in order to begin the survey, after which a series of survey questions appeared.

Survey takers were not pressured to take the survey by any means. They took the survey voluntarily. And the responses were recorded automatically in the e-cloud keeping the recipients anonymous.

\section{Result}

Amongst the 150 survey takers aged between $12-30,54 \%$ were female and $46 \%$ were male. There were 122 respondents belonging to the age group from 20 - 25 years i.e. $81 \%$ of the total survey takers and $3 \%$ i.e. 5 respondents were in between (15-20). And the remaining 23 respondents belonging from the age group (25-30). 


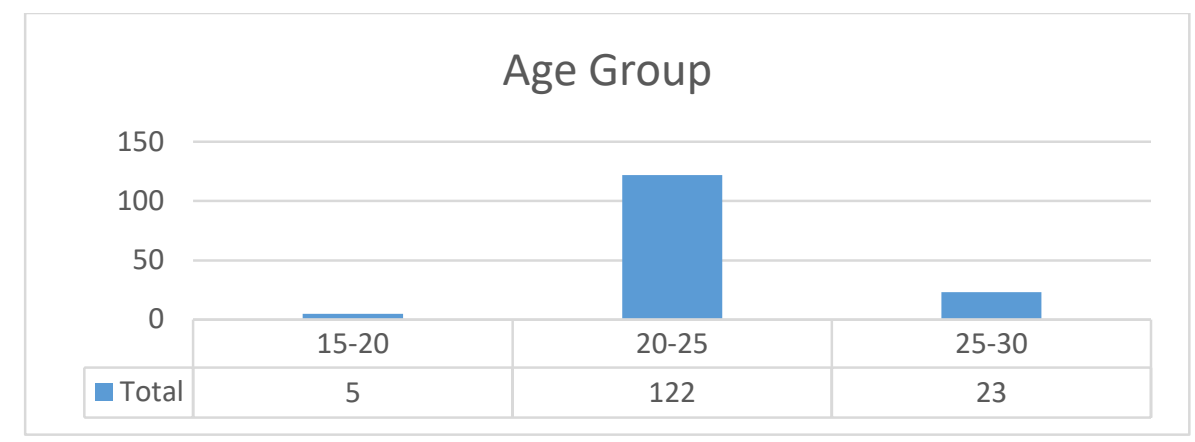

Figure 5. Age Group of Survey takers

Besides, $8 \%$ of them were seen to have OCD and were extremely serious about washing hands frequently. Up to 30 times a day.

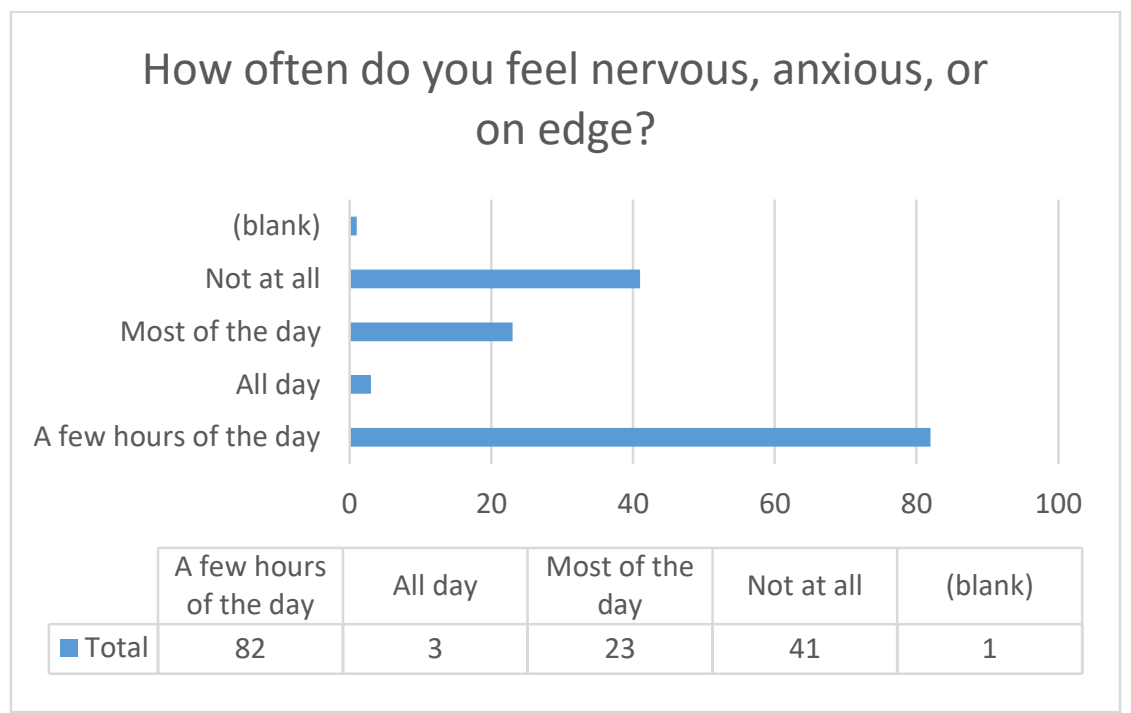

Figure 6. Anxiety/Stress level

$45 \%$ were feeling neutral on the day they took the survey, $27 \%$ felt good, almost $19 \%$ felt bad, and $6 \%$ felt really good. $55 \%$ of them remain stressed or anxious a few hours of the day, nearly $28 \%$ didn't feel stressed or anxious at all and 15\% felt anxious and restless most of the day. 


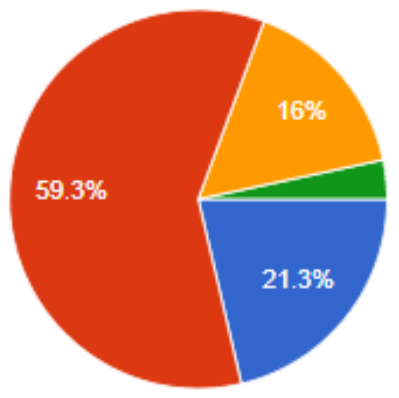

Figure 7. Stress related to Covid-19

$59 \%$ of youngsters stress about Covid-19 few hrs. Of the day, $21 \%$ didn't worry about the virus at all and $3 \%$ worried about it throughout the day. $96 \%$ of the survey respondents had not been corona infected but unfortunately the rest, i.e. $4 \%$ were tested corona positive and have recovered by now. And majority candidates have corona positive relatives, for whom they feel sorry about.

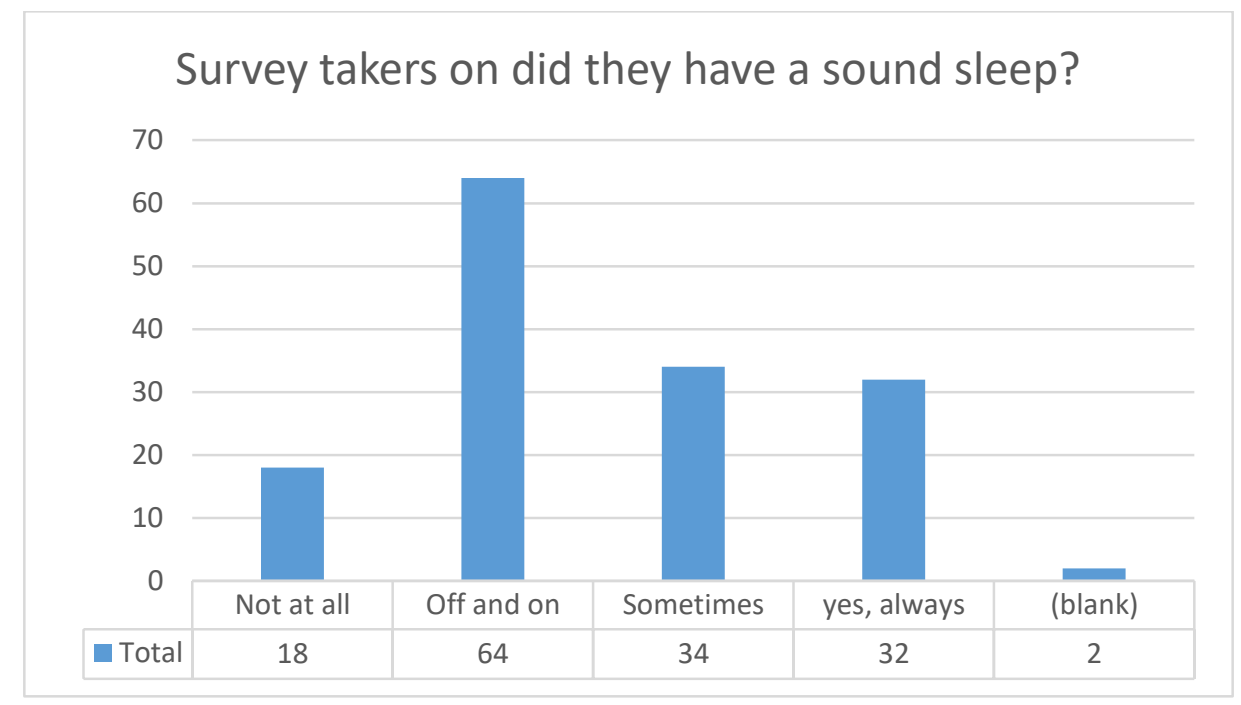

Figure 8. Sleep quality

$66 \%$ of them face difficulty to have a sound sleep and $21 \%$ of them always have a disturbed sleep. 


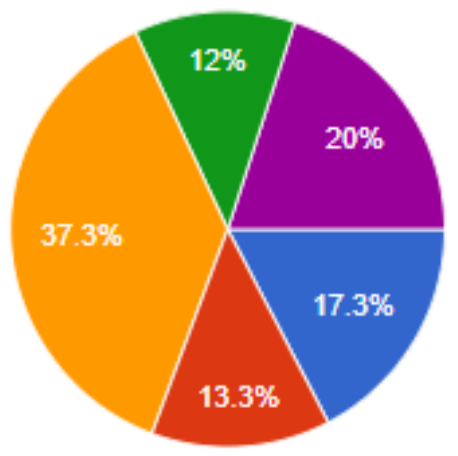

Sleep more than I used to

Sleep less than I used to

Sleep during day, stay awake during night

No change

Have trouble sleeping when I want to

Figure 9. Change in sleeping pattern

Stress can unfavorably affect sleep quality and duration, while insufficient sleep can increase stress levels. Both stress and sleep deficiency can lead to lasting physical and mental health problems. It is crucial that people who are having issues with stress or lack of sleep do not try to tackle these problems alone [18].

Maximum of the survey takers, sleep during day time and cannot sleep during the night that is $37 \% .13 \%$ are sleeping less than they used to and $17 \%$ are sleeping more than they used to and $12 \%$ are not facing any change in their sleeping cycle.

Not getting enough sleep can weaken our body's immune system and lead to both weight gain and loss. When one does not get enough sleep, increase in certain health risks such as, cancer, diabetes, and many accident can take place due to lack of concentration.

$65 \%$ of were found to be restless at times which triggers anxiety in them, nearly $21 \%$ do not have any restlessness, nearly $11 \%$ feel restless all the time and $3 \%$ need medication to feel better. $55 \%$ agree to get irritated easily, nearly $19 \%$ get irritated easily but not because of the pandemic, $15 \%$ never get irritated and nearly $11 \%$ remain irritated all the time. 


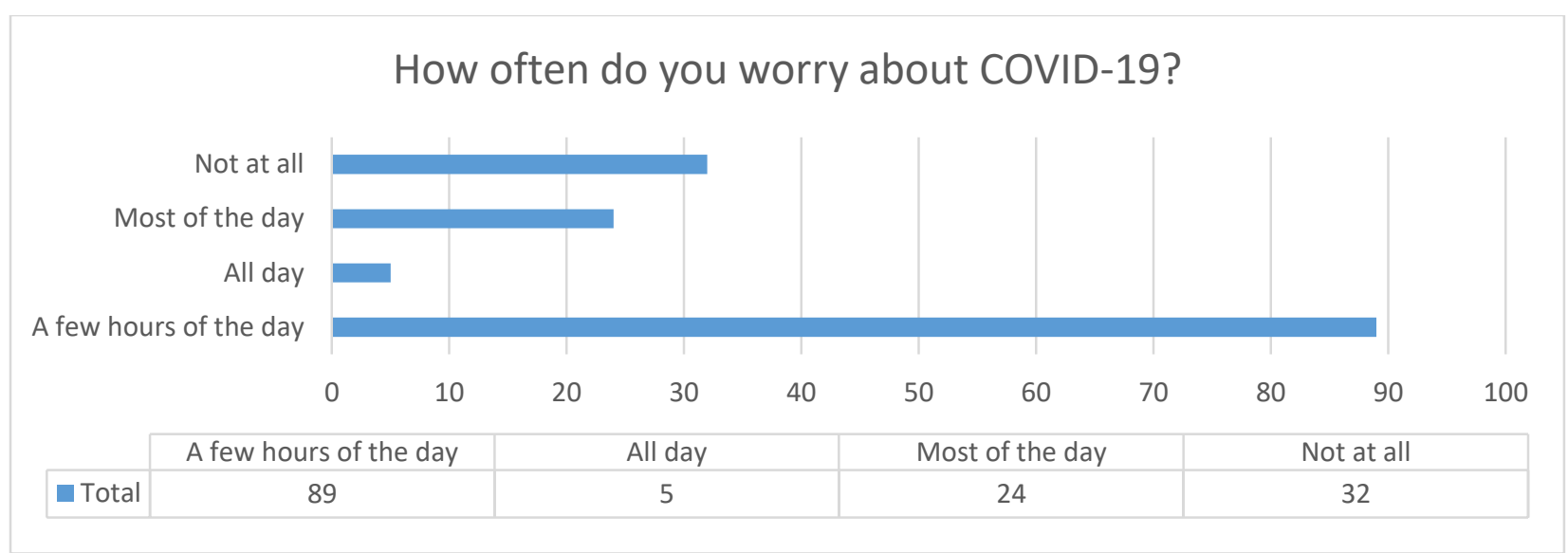

Figure 10. Stress related to Covid-19

Nearly $69 \%$ are sometimes in fear that something bad might happen to them. $20 \%$ are scared of being affected by the deadly virus and $12 \%$ are not threatened by the virus. Nearly $41 \%$ understand the importance of staying indoors, nearly $19 \%$ are feeling bad about not being able to go to their workplace or education institution.13\% feel really bad, nearly 13\% are heartbroken, not being able to go out and nearly $9 \%$ find it average and 6\% feel great about not going out. $100 \%$ of the survey takers think mental health is treated lightly in our country.

Is your family facing a financial crisis due to the pandemic?

150 responses

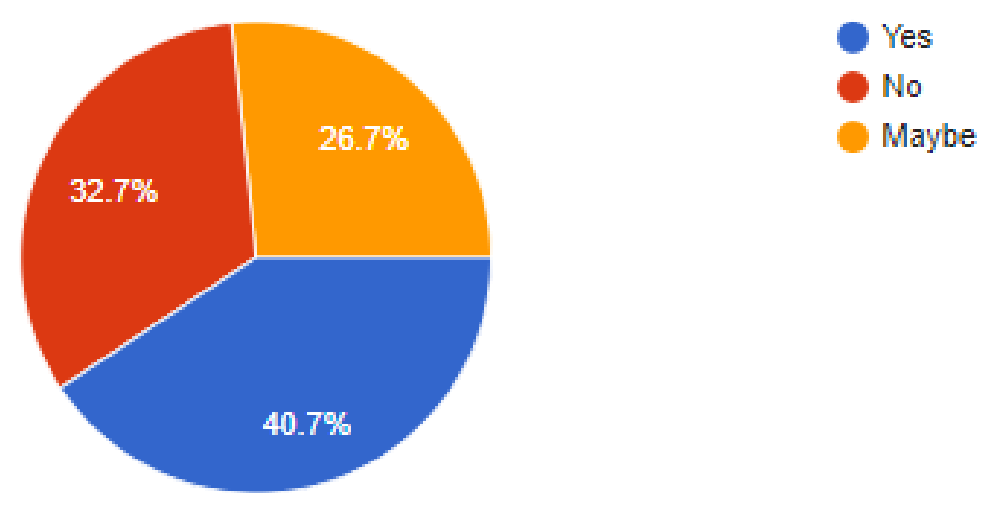

Figure 11. Financial crisis status of respondents 
Amongst the participants, nearly $41 \%$ agree on facing a financial crisis during the pandemic and almost $27 \%$ are unsure whether they are having financial difficulties or not. The rest are doing as they were before, which is nearly $33 \%$.

As mentioned earlier, $22 \%$ are currently a victim of domestic violence or seeing someone face domestic violence in their own household. Almost 3\% didn't want to answer this question and $75 \%$ are not facing or seeing anyone face domestic violence in their household.

$34 \%$ of the survey takers spend 2-4 hrs. a day, browsing apps, watching videos on YouTube, Facebook, and other apps, 33\% of them spend 4-8 hrs. Per day browsing the internet. And 32\% spend more than 10 hrs. Per day browsing the internet. 90\% can differentiate between facts and fake posts/news in social media and $10 \%$ cannot.

\section{Do you workout/yoga/walk regularly?}

150 responses

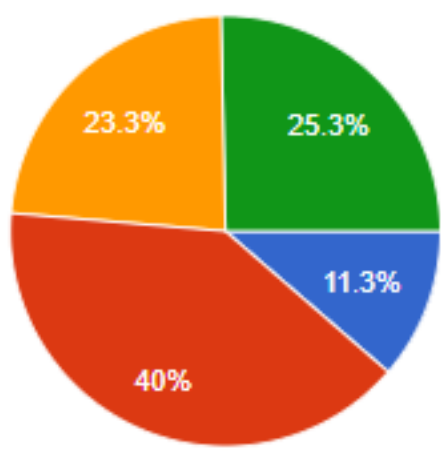

Everyday

Occasionally

Never

Some days a week

Figure 12. Physical exertion status

$40 \%$ of them exercise, walk, or perform yoga occasionally, $25 \%$ exercise some days a week, and $23 \%$ of them never engage in any physical exertion and $11 \%$ workout and exercise every day. $61 \%$ often think they do not have anyone to talk to, $20 \%$ always think they are alone and have no one to talk to when they are restless or worried, and nearly $19 \%$ never feel that way. $44 \%$ feels irritated and anxious about wearing masks and gloves, $31 \%$ sometimes feel irritated and nearly $25 \%$ find it ok to wear gloves and masks. About $46 \%$ of them stay laid in bed throughout the day, nearly $23 \%$ 
work actively, $16 \%$ remain seated in a bed most of the time and $16 \%$ spend most of the time seated in bed.80\% are currently attending class or working from home and nearly $7 \%$ remain idle all day, the rest are involved in other works. Almost 37\% of them remain in front of screens for 2-3 hrs. Almost $32 \%$ for $4-8$ hrs. $17 \%$ for less than an hr. $7 \%$ for $12+$ hrs. Each day.

\section{Do you face any difficulties after hrs. in front of the screen? ( headache, back pain, etc)} 150 responses
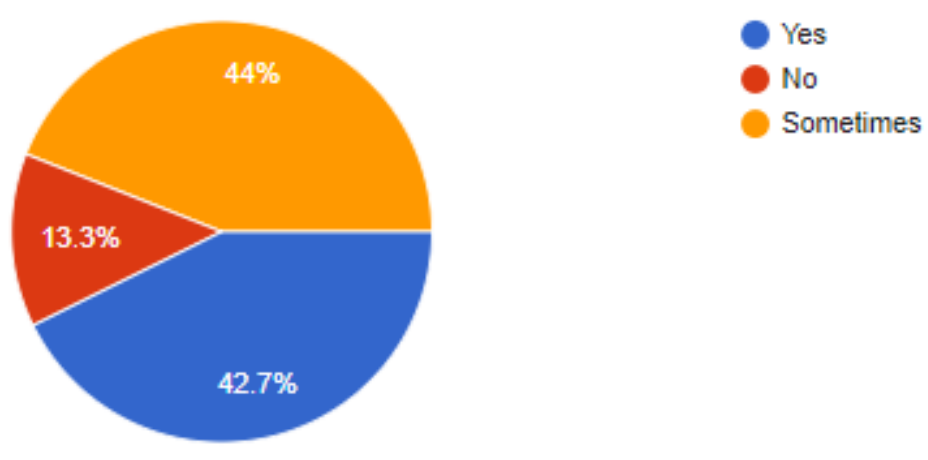

Figure 13. Difficulties faced while sitting for hrs.

And nearly 75 for 10-12 hrs. each day. $86 \%$ face difficulties like headache, back pain etc. after hrs. in front of the screen, $13 \%$ feel they don't face any problem. The saddest part is nearly $49 \%$ sometimes think their life is meaningless and don't know what to do next, nearly $28 \%$ often think their life is meaningless and nearly $30 \%$ never feel this way.

\section{Anxiety Leading to Depression}

Mental health is a level of psychological well-being. It is the "psychological state of someone who is functioning at a satisfactory level of emotional and behavioral adjustment" (Libretexts, 2021). Including our emotional, psychological, and social well-being, affecting how we think, feel, and act. It also helps to determine how we handle stress or make choices. Mental health is very essential at every stage of life, from childhood and adolescence through adulthood. 
Many people struggle with mental health problems but find it difficult to address their concerns because they don't really know how to define what's happening in their minds or if the listener will actively understand and accept their thoughts.

Based on the questionnaire completed, survey respondents were more likely to experience anxiety and were persistently thinking regarding COVID-19. They felt uncertain, fearful and isolated during current times. It was also shown that they experienced disturbed sleep, poor appetite, agitation, separation-related anxiety, and sadness for not being able to socialize. Similarly, wearing protective gears like mask, gloves are considered irritating to many.

Similarly, effective physical activity can promote feelings of vitality. Importantly, even low to moderate volume and low to moderate-intensity exercise seems to be beneficial. As less as $10 \mathrm{~min}$ moderate-intensity walking can improve mood. But, from the data collected, not many young people are involved in daily physical exertion or walking. Maximum survey takers either workout or go for walk occasionally and $23 \%$ never perform any physical activity to keep them fit.

Human beings are the most important component of the Environment and Youth being the most strong and efficient component of the human beings can change the future of the environment with their well-being and positive decision making. But the global pandemic has put everything on stop for months causing stress and anxiety amongst the quarantined people.

Young people being cut off from the love, support, and close contact of family and friends can contribute to poor mental health. It is an illness that involves the body, mood, and thoughts. Clinical depression affects the way one eats and sleeps. It affects the way one feels about themself and those around them.

Months of social distancing and sheltering at home can leave someone feeling isolated and lonely, having to deal with problems all alone. With all the fear and uncertainty regarding COVID-19, it is natural to feel tense. But, when the worries spiral out of control they can lead to panic and anxiety. Since anxiety and depression are considered to stem from the same biological vulnerability, one can often lead to other disorders such as lack of proper decision making, insomnia, preoccupation with death, fatigue, weight fluctuations, weekend immunity system, increased pain sensitivity and many more. 
Considering current situations, not only the ones who are isolated in stress and depression, the financial crisis is another great cause. Parents not being able to provide better facilities, internet, and smart devices to their children are themselves in a depression not being able to meet their children's needs in such crisis.

Similarly, experiencing major changes in life, such as the death of a loved one, the loss of a job, being Covid19 positive, or dealing with relationship difficulties, can bring overwhelming levels of stress. Experiencing multiple stressors at once can make one more vulnerable to depression.

Moreover, young people being bored and lonely can make unwise decisions and may choose unhealthy ways of coping to deal with stress. Like overeating junk food, consumption of marijuana or other drugs to feel less stressed. While these methods may provide brief relief, eventually it will lead to severe depression ruining the body's immunity system.

\section{Effects on Individuals with Pre-Existing Psychiatric Illness}

Mentally challenged patients are substantially more likely to develop infectious diseases, such as lung diseases and are at considerable threat of experiencing more negative physical as well as psychological effects during a potentially fatal pandemic, the Covid-19 (Chiang et al., 2019) [23].

These conditions also trigger an outburst of temper tantrums in mentally challenged people and conflict between parents and adolescents. Although prior to the pandemic, they had been facing difficulties even while attending special institutions, but in due course, they had learnt to develop a schedule to adhere to for most of the time of the day (APA, 2020; Cortese et al., 2020; UNICEF, 2020a)[14]. To cater to these challenges, it is difficult for parents to handle the challenged children and adolescents on their own, as they lack professional expertise and they mostly relied on schools and therapists to help them out (Dalton et al., 2020).

Cognitive decline, poor awareness levels, impaired risk perception, and reduced concern about personal hygiene can increase the chances of acquiring infectious diseases. Moreover, social 
perception against mental sickness makes the management of patients with COVID-19 more challenging.

Similarly, based on the study it is found that for individuals with Obsessive-Compulsive Disorders (OCD) hand washing goes well beyond a concern with cleanliness they practice frequent hand wash. 2-3 times an hour and even 30 times a day.

Hand-washing being an anchor precaution to prevent COVID-19 transmission adds further to the misery of a known washer OCD patient. Those with hand-washing compulsions are obsessed with the fear of contamination and often wash their hands repeatedly until they are chapped and sometimes even bleeding. They may also ritualize the process, for example, by washing each finger individually and in a specific order. An interruption to the ritual might compel starting over and over from the beginning [15].

Side by side, anyone taking the treatment had to cancel all the counselling or medications because of the nationwide lockdown. Which might have a negative effect as well. Moreover, embarrassment often causes people to suffer from OCD for years before seeking help and to try to keep their behavior out of sight even from their close ones.

\section{Struggles of a sudden shift to Remote Learning on health}

The pandemic is forcing global experimentation with remote teaching. There are many signs that this crisis is going to transform many aspects of life. If remote teaching proves to be a success, Education could be one of them.

Online Learning might get stressful and irritating at times. Some are still struggling to keep up with the monotonous indoor routine each day. It is adding more to the existing stress with all that is taking place for the past couple of months. 


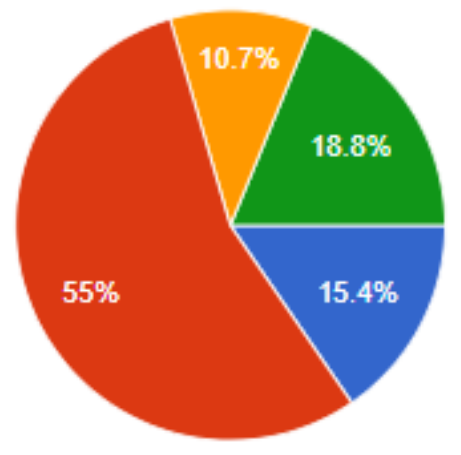

Not at all

Sometimes

All the time

Yes, but not because of the pandemic

Figure 1. Impact of monotonous lifestyle

Moreover, distance learning has led to more out-of-pocket spending by our nation's teachers and parents. Unfortunately, families without sufficient smart resources and access to reliable internet may have severe stress on how to continue virtual learning with limited income. As they are worried about their adolescents falling behind and thrive to give their children a better education even under such circumstances.

On the other hand, parents with mentally challenged children or adolescents are facing a different level of stress. As they cannot take them for therapies or face to face interactive classes, they tend to get hyper and annoyed quite frequently.

Other than that, young and middle-aged people are spending more hours focusing on digital screens, their eyes are going through an exhausting endurance workout. Eye strain from hours of screen time can result in eye irritation, fatigue or blurred vision, back pain and headache, are very common, according to the survey conducted. $44 \%$ survey takers mentioned facing difficulties as such.

Doctors from research have linked screen time with an increased amount of sedentary behavior in children and teens [24]. While there is no long term evidence yet to link screen time to an increased 
risk of health conditions like cardiovascular disease and high cholesterol, there is mounting evidence that it is associated with obesity.

\title{
Increased usage of internet and smart devices
}

\author{
Involvement in activities during the lockdown
}

How long do you spend on apps like YouTube, WhatsApp, Facebook, Instagram, Twitter,

Snapchat, Tiktok on a daily basis?

150 responses

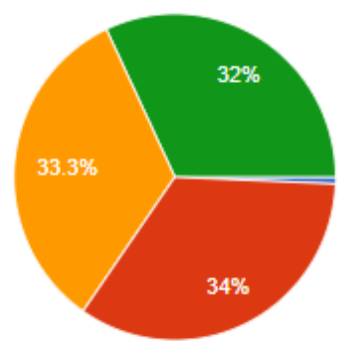

Not at all

2-4 hrs a day

4-8 hrs a day

More than 10 hrs a day

Figure 2. Time spent online for leisure purpose

How long do you sit in front of screens to attend zoom/skype meetings or classes or for selfstudy?

149 responses

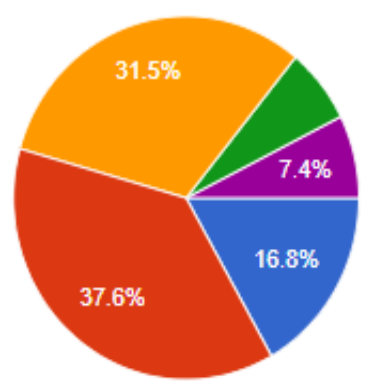

less than an hour

2-3 hrs

4-8 hrs

- $10-12$ hrs

$12+$ hrs each day

Figure 3. Time spent online for class/work purpose 
From the study conducted, it is seen $32 \%$ survey respondents spend more than $10 \mathrm{hrs}$. each day, using applications on the internet. And $7.4 \%$ spend more than $12 \mathrm{hrs}$. each day attending online conferencing calls.

In a study on U.S. adults (Madhav et al., 2017) it was found spending six hours or more a day in watching TV or using computers was associated with a higher risk for depression. Another study (Heid, 2018) published in 2018 shows, young people who spend seven hours or more a day interacting with screens are twice as likely to be diagnosed with depression or anxiety as those who use screens more moderately. Similarly, a study of 2018 (Credits Michele W. Berger Writer et al., 1970) from the University of Pennsylvania found students who trimmed their use of social media to 30 minutes a day experienced significant improvements in wellbeing.

Comparing these results with the study conducted, it is indeed saddening that the youth of our country are pretty much in threat of depression and computer vision syndrome. CVS is a problem that has emerged in this century following increase usage of a computer. There is a correlation between ocular symptoms such as pain, redness, dryness, blurring of vision, double vision and other head and neck sprains and computer usage. Sitting in an incorrect posture for hours can further cause headache, nausea, back pain and can create a tendency to get agitated easily.

\section{Substance abuse during lockdown}

Mental health problems can be directly linked with substance abuse amongst the young generation. Studies show issues like depression, developmental lags, conduct problems, personality disorders, suicidal thoughts, and other psychosocial dysfunctions are some mental health barriers caused by use of drugs like Marijuana which is prevalent amongst youths.

Marijuana use, has been shown to interfere with short-term memory, learning, and psychomotor skills. Motivation and psychosexual/emotional development may also be influenced (Bureau of Justice Statistics, 1992) [22]. 
From the findings of the survey conducted, nearly 5\% survey respondents mentioned their involvement with Cannabis consumption in their leisure time or to relieve stress. Considering that some of the respondents might have obscured the reality, the rate is still very alarming as the survey was conducted on young educated students from well to do families.

\section{Impact of rising unemployment on mental health}

The global pandemic has brought greater layoffs and a rise in the unemployment rate. Certain industries have been hit harder than others, such as the leisure and hospitality sector.

According to a survey titled "Coping with COVID -19 and Individual Responses: Findings from a Large Online Survey" was conducted on 30,000 people, covering all divisions and districts. The survey was conducted by the Bangladesh Institute of Development Studies (BIDS). It was later unveiled at a virtual conversation titled "In the Shadow of COVID - Coping, Adjustments, Responses".

A noticeable negative impact was seen in the findings. As per the report, $19.23 \%$ of participants with income less than 5,000 takas reported that their income was reduced by $75 \%$, while $23.31 \%$ participants with income between 5000-15000 taka reported an income reduction by 50\% relative to last month's income.

Similarly, SME entrepreneurs in the rural areas are affected the hardest due to the impact of the global shutdown, as their revenue has dropped by $67 \%$ in 2020 compared to that of 2019 .

An article from a reputed daily of Bangladesh [28] states that family income has fallen $74 \%$ between March and May and over 1.4 million migrant workers have lost jobs because of the pandemic. It is indeed a matter of great concern on how the families are surviving. It is quite evident that things might get suffocating at times. With each passing day, anyone might get quieter and more anxious as the number of coronavirus patients, the rising number of deaths, shutdown getting extended, cut down in salary, and even unemployment. 
Whether it's temporary or permanent, unemployment can lead to stress, anxiety, depression and other mental health challenges. In the current situation, there is a much lower chance of the unemployed landing a new job anytime soon. And it's uncertain when social distancing measures will terminate or how the economy will be when they are able to return to work. The inability of less or no income or not being able to leave home for work adds an increased risk of mental health issues.

The loss of income could further lead to a decline in the standard of living of the individual or household. With increasing costs, being unemployed for too long might reduce their lifestyle drastically which, of course, could influence both the physical and mental health of everyone in the family. Economic insecurity might further lead the main earning source of the family to anxiety, loss of self-esteem, and depression.

\section{A rise in Domestic Violence}

The emotional stress people are going through are impacting largely on their mental health and that often leads to several things including domestic violence.

Are you or anyone you know facing domestic violence during this pandemic?

150 responses

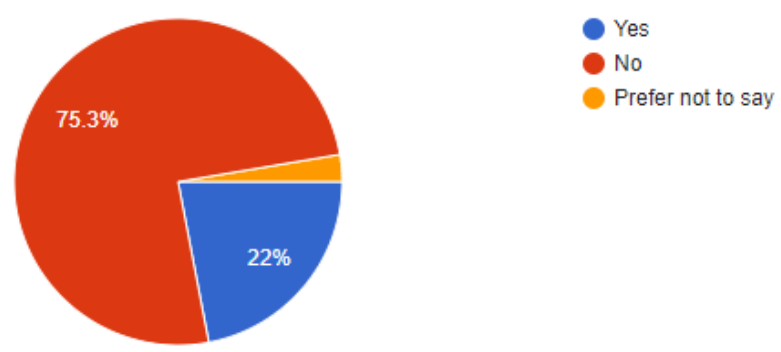

Figure: 4 -Domestic Violence during pandemic 
Violence against women and girls has always been a social, cultural and economic problem in Bangladesh. According to an article [13], violence against women and children is on the rise in Bangladesh amid the COVID-19 lockdown. Experts say lack of social activities and financial pressures are increasing cracks within families.

Considering the rising domestic violence and killing of women, by their own husband or known ones, rights activists say the lockdown has made women and children more vulnerable to domestic violence and abuse as many of them are confined to their homes with no outside support.

According to an article by Manusher Jonno Foundation (MJF) published on June, 2020, a survey was conducted on at least 4,249 women and 456 children were subjected to domestic violence in 27 out of 64 districts of Bangladesh in April, with 1,672 women and 424 children facing violence for the first time in their lives. Women, facing domestic violence blame the frustration of their husbands caused by lockdown for their family's unrest. From the same survey, it shows that, amongst 17,000 women and children, 848 women were tortured physically, 2,008 mentally, 85 sexually, and some 1,308 faced financial restrictions from their husbands. The survey also said that four women were raped, one killed, and 20 women were sexually assaulted by people not related [29].

Seeing the murder and violence cases on women and children all across Bangladesh, Rights activists say the lockdown has made them more vulnerable to domestic violence and abuse as many of them are confined to their homes with no outside support.

Shockingly, $22 \%$ of the people surveyed for this research project, are currently facing or seeing someone face domestic violence in their own household. Mental health effects of violence against men or women can include behavioral problems, sleeping and eating disorders, depression, anxiety, posttraumatic stress disorder (PTSD), self-harm and suicide attempts, poor self-esteem, harmful alcohol, and substance use.

Similarly, after completion of the survey data required for this paper, the result showed that nearly $23 \%$ of the survey takers found their life meaningless most of the time. And nearly $29 \%$ sometimes felt their lives to be meaningless. Considering such data from educated families and university going adults is intolerable. 


\section{Discussion}

This study investigated the mental health of the young population, mostly college and university going students in Bangladesh during the COVID-19 pandemic. This study provides data on higher

levels of anxiety, stress, depressive symptoms, and change in practices during the COVID-19 epidemic.

Findings from the study indicate that COVID-19 pandemic was associated with increased mental and physical health issues. Individuals already dealing with mental health issues like OCD are stressing more over keeping themselves sterilized. Though the majority of the survey takers had been Corona negative till now 55\% are stressed thinking about COVID-19 each day. Seeing close relatives and friends suffer and the guilt of not being able to help is demoralizing many. The majority had a drastic change in their sleeping and eating habits as well. Sleep is often neglected by young ones but its importance is vital as it enables the body to repair and be fit and ready for another day. Not getting adequate rest may also lead to excess weight gain, heart disease, indigestion and increased illness duration.

Not working out maybe an addition to their current unhealthy lifestyle. The majority have a tendency of getting restless or angry quite frequently. Though the educational institutions or working places are physically closed, students or work holders have to spend hours in front of the screen completing their tasks. It is not only affecting them mentally but also physically. Back pain, strain in the eyes are the most common difficulties youngsters are dealing with. Not having adequate smart devices and internet facilities for the parents as well their children may be another cause of stress and depression.

The COVID-19 crisis has brought Bangladesh to the risk of massive economic fallout. Unemployment is spiking overnight and is causing mental breakdown for many. With frustrations and anxiety building at the household level, it is now crucial to assess our country's rising cases on domestic violence and sexual abuse. Women and children are not safe and can be exploited anywhere. Statistics show sexual violence in Bangladesh nearly doubled and took in Bangladesh due to the lockdown. 
Surprisingly, respondents belonging from well off and educated families are facing financial crises which also lead to domestic violence. $22 \%$ that is, 33 from the 150 survey takers are currently facing or seeing someone face domestic violence in their own household, which needs to be brought to light.

\section{Limitations}

Though the global pandemic is residing amongst us for months now, it is still not identified completely. There are limited studies and findings for the Covid-19 crisis. Even though many people are affected with mental illness, there is a strong social stigma attached to mental ill-health, and maybe not everyone taking the survey opened up completely about their mental health. And to maintain confidentiality, contact details and personal information from the respondents were not collected as a result it was not possible to distinguish between their pre-existing mental health symptoms (if any) and new ones.

Since Bangladesh was under a nationwide lockdown from the $26^{\text {th }}$ of March, 2020, taking personal interviews was not possible thus the data had to be collected online.

Therefore collecting data from individuals without access to the internet were not possible and a particular group with a similar background could take the survey. False data might be added by the survey takers to avoid conflicts, other personal issues or just to save time. Collecting resources and data online was another challenge as many publishers do not provide open-access to their research. Thus, finding relevant materials online was another limitation.

\section{Opportunities}

The study can be precise if carried in the general population and involving people from different backgrounds. Since educated, middle class or upper middle class youngsters were surveyed, a better research can be conducted if youth from all classes living in Dhaka city or Bangladesh are surveyed. More age groups can be used for a better comparison, identifying which group is more 
vulnerable and why. More relevant literature and research studies on these topics can provide further recommendations and solutions.

Similarly, opening a platform where everyone can share their findings related to similar topics might be fruitful for further studies. As many researchers may conduct similar surveys and compare them according to regions or other perspectives.

\section{Recommendations}

The adolescent phase is a crucial period for developing and maintaining social and emotional habits important for mental and physical well-being. These include adopting healthy sleep patterns, taking regular exercise; developing coping, problem-solving, and interpersonal skills; and learning to manage emotions. Supportive environments in the family, college, university or work place and in the wider community are also important.

An estimated 10-20\% of adolescents globally experience mental health condition, yet these remain underdiagnosed and undertreated. The more risk factors youth are exposed to, the greater the potential impact on their health.

In Bangladesh, the psychological impact of COVID 19 pandemic seems to affect significantly to the younger population. A series of recommendations concentrating on health care of the youth, during the preparedness, outbreak and post-outbreak period of COVID 19, should be considered as not much is not known about when we can go back to our older lifestyle. More focus should be given on enriching the knowledge as fear and panic occur due to limited knowledge or poor adaptability. Knowing less or following misconceptions can cause severe and unrepairable loss.

To increase preparedness amongst the youth, specialist team on mental health aiming to provide social support, online service, psychotherapeutic interventions and monitoring and training of the subordinates subsequently could be helpful. According to WHO, suicide is the third leading cause of death amongst the youth, belonging from the most emotional age group. If they get proper counselling and moral support is from family, peers or known ones, they might not feel lonely and isolated in such times. 
It is fine to might feel low, bored or stressed at times. Though many are facing financial crisis now, it will get better with time. But there's a difference between changing of mood through healthy alternatives and turning to drugs or alcohol. We know an idle mind is the devil's workshop, drugs or alcohol can seem like an acceptable choice and the quickest fix. Therefore keeping oneself busy is extremely crucial. It can as well lead to a destructive path towards addiction, especially when the age group is vulnerable.

A healthy nutrition, access to support, limited browsing on social media, avoiding unnecessary thinking and not stressing over news is recommended. Regular physical exercise is needed to boost the mental and physical health. Strong, positive energy coupled with the right attitude is important to cope with stress and boredom while staying home. A productive routine should be set to keep mind and body fresh. Physical activity has the potential to ward off detrimental cardio metabolic effects of inactivity and to reinforce psychological resources and coping skills.

Physicians and other health care professionals should use this time as a window of opportunity to provide physical activity counselling to their patients especially to consider the mental and physical health of the youth in such times. As they are more emotionally vulnerable compared to the older or younger age groups.

From the data collected, many are seen suffering from lack of sleep and change in sleeping patterns during the lockdown leading to anxiety causing frequent anger and restlessness. Even though, it is recommended by physicians to work out or go through physical exertion for at least 10 mins per day, many survey takers responded they do not have daily exertions, which may be causing such restlessness. Though going out in crowd is still discouraged to minimize the spread of the ongoing pandemic, one can still go out in open air wearing protective gears and only in less crowded places.

If not, one can simply walk up and down the stairs of their house or carryout equipment free exercises indoors. Similarly, utilizing the brain and body in positive and productive work like gardening, cooking, painting, or anything interesting could be done to relax the mind.

Moreover, as the world is going all virtual, sitting in front of computers and other electric devices has become a necessity. Minimizing the use is vital in such circumstances. Only attending important calls and classes could be done, giving the eyes and back less stress. 
Lastly, violence against women and children in Bangladesh has reached epidemic proportions. According to statistics, it has doubled during the lockdown. It can happen to anyone, no matter age, race, or education qualification. If someone is experiencing domestic violence, it needs to be listened without any judgment and needs to be helped out from that state.

During the survey, it is seen 33 out of 150 survey takers are currently facing or seeing someone face domestic violence in their household. It is a very big group and something needs to be done to help them. If someone is in fear that the violence may continue, it is essential to be available for them. If they do not want to leave that place, giving them assurance of help could boost them and give them confidence to deal with the situation.

\section{Conclusion}

The lockdown implemented in an attempt to contain the SARS-CoV-2 virus is unprecedented for most. It may take weeks or months to accept the new normal, and managing this impact requires self-dedication and effort as well as a change in certain practices.

The findings from this study indicates that the widespread pandemic causing rise in unemployment, domestic violence, substance abuse, remote learning and several other reasons is severely impacting our mental health. With outcomes such as anxiety, stress, and change in eating /sleeping habits, depression, and anger.

It appears that the COVID-19 pandemic has led to a vigorous and multifaceted response with several health risks. Mental health being considered to be a folk's tale amongst many, is undoubtedly being taken into consideration these days. As a certain population is now aware and have learnt about prioritizing mental health. Therefore, physicians and other health care professionals should be more careful in counseling and guiding the vulnerable age groups.

In spite of all the trauma and negative affects the pandemic has put us through, there is some hope that mental health issues and domestic violence cases often neglected in less developed countries can now get an increased availability of support services due to more ongoing research and investigation on these topics. 


\section{Bibliography}

1. SL. Lustig, M., Shaw, J., M. Fazel, J., M. Hodes, D., T. Bean, I., EB. Wiese, I., . . Steiner, H. (1970, January 01). Mental health issues in unaccompanied refugee minors. Retrieved September 09, 2020, from https://link.springer.com/article/10.1186/1753-2000-3-13

2. Adolescents and mental health. (2017, April 09). Retrieved September 09, 2020, from https://www.who.int/maternal_child_adolescent/topics/adolescence/mental_health/en/

3. Unb. (2020, June 25). 13pc people lost jobs due to Covid-19 pandemic: BIDS survey. Retrieved September 09, 2020, from https://www.thedailystar.net/business/13pc-peoplelost-jobs-in-bangladesh-due-covid-19-pandemic-1920309

4. Banna, H. A., Sayeed, A., Kundu, S., Christopher, E., Hasan, M. T., Begum, M. R., . . . Khan, S. I. (2020). The impact of the COVID-19 pandemic on the mental health of the adult population in Bangladesh: A nationwide cross-sectional study. doi:10.31234/osf.io/chw5d

5. Welch, A. (2018, August 06). Health experts say parents need to drastically cut kids' screen time. Retrieved September 09, 2020, from https://www.cbsnews.com/news/parents-needto-drastically-cut-kids-screen-time-devices-american-heart-association/

6. Community, T. (2020, September 04). Novel Coronavirus Information Center. Retrieved September 09, 2020, from https://www.elsevier.com/connect/coronavirus-informationcenter

7. Outbreak Investigation: Mental Health in the Time of Coronavirus (COVID-19). (n.d.). Retrieved September 09, 2020, from https://www.frontiersin.org/researchtopics/13706/outbreak-investigation-mental-health-in-the-time-of-coronavirus-covid-19

8. Hossain, K. A., Roy, S., Ullah, M. M., Kabir, R., \& Arafat, S. Y. (2020). COVID-19 and Mental Health Challenges in Bangladesh. Advances in Medical, Dental and Health Sciences, 3(2), 31-33. doi:10.5530/amdhs.2020.2.8

9. Mental health and COVID-19. (2020). doi:10.3310/collection_40756

10. Newby, J. M., O’Moore, K., Tang, S., Christensen, H., \& Faasse, K. (2020). Acute mental health responses during the COVID-19 pandemic in Australia. Plos One, 15(7). doi:10.1371/journal.pone.0236562 
11. Uddin, M., Alam, M. T., Ahmed, H., Mohammad Khan, N., \& Talukder, S. (2020). Mental Health and Psychosocial Support (MHPSS) in COVID-19 Outbreak. Bangladesh Journal of Infectious Diseases, 7, S45-S47. https://doi.org/10.3329/bjid.v7i0.46801

12. Nirmita Panchal, R., \& 2020, A. (2020, August 21). The Implications of COVID-19 for Mental Health and Substance Use. Retrieved September 25, 2020, from https://www.kff.org/coronavirus-covid-19/issue-brief/the-implications-of-covid-19-formental-health-and-substance-use/

13. (www.dw.com), D. (n.d.). COVID-19 lockdown increases domestic violence in Bangladesh: DW: 12.05.2020. Retrieved September 25, 2020, from https://www.dw.com/en/covid-19-lockdown-increases-domestic-violence-inbangladesh/a-53411507

14. Singh, S., Roy, D., Sinha, K., Parveen, S., Sharma, G., \& Joshi, G. (2020, August 24). Impact of COVID-19 and lockdown on mental health of children and adolescents: A narrative review with recommendations. Retrieved September 26, 2020, from https://www.sciencedirect.com/science/article/pii/S016517812031725X

15. Rosenberg, F. (2017, February 02). How Much Hand Washing Is Too Much? OCD Washing Hands. Retrieved September 26, 2020, from https://www.anxiety.org/doesexcessive-hand-washing-mean-obsessive-compulsive-disorder

16. B. Nussbaumer-Streit, V., E. Lowther, M., SK. Brooks, R., KL. Piercy, R., R. Guthold, G., KA. Bowden Davies, S., . . Nieman, D. (1970, January 01). Physical activity during COVID-19 induced lockdown: Recommendations. Retrieved September 26, 2020, from https://occup-med.biomedcentral.com/articles/10.1186/s12995-020-00278-9

17. Weber, M. (n.d.). Dealing with Depression During Coronavirus. Retrieved September 26, 2020, from https://www.helpguide.org/articles/depression/dealing-with-depressionduring-coronavirus.htm

18. Stress and sleep: What's the link? (n.d.). Retrieved September 28, 2020, from https://www.medicalnewstoday.com/articles/322994 
19. Libretexts. (2021, April 30). 2: Psychological Health. Medicine LibreTexts. https://med.libretexts.org/Bookshelves/Health/Book\%3A_Health_Education_(Rienk_and _Lundin)/02\%3A_Psychological_Health.

20. Amit S. Coronavirus and impact on the Bangladesh economy: Assessing the damage of the black swan of 2020. Asia-Pacific Journal Japan Focus. 2020

21. World Health Organization. Coronavirus disease (COVID-19) pandemic. 2020 [cited 03 May 2020]. https://www.who.int/emergencies/diseases/novel-coronavirus-2019

22. American Psychological Association. (n.d.). Substance use during the pandemic. Monitor on Psychology. https://www.apa.org/monitor/2021/03/substance-use-pandemic.

23. Chiang, C.-H., Tsai, M.-C., Ng, Y.-Y., \& Wu, S.-C. (2019, October 11). Mental Disabilities Increase the Risk of Respiratory Infection-related Healthcare Utilization. International journal of environmental research and public health. https://www.ncbi.nlm.nih.gov/pmc/articles/PMC6843153/.

24. ScienceDaily. (2021, March 5). Research identifies impact of teenage screen use. ScienceDaily. https://www.sciencedaily.com/releases/2021/03/210305113449.htm.

25. Madhav, K. C., Sherchand, S. P., \& Sherchan, S. (2017, August 16). Association between screen time and depression among US adults. Preventive medicine reports. https://www.ncbi.nlm.nih.gov/pmc/articles/PMC5574844/.

26. Heid, M. (2018, October 29). There's Worrying New Research About Kids' Screen Time and Their Mental Health. Time. https://time.com/5437607/smartphones-teens-mentalhealth/.

27. Credits Michele W. Berger Writer, Credits Michele W. Berger Writer, Credits, Michele W. Berger Writer, Berger, M. W., Writer, Date November 9, Date, 9, N., Subtopics Psychology, Subtopics, Psychology, Schools School of Arts \& Sciences, Schools, Sciences, S. of A. \&, Share Twitter Facebook, Share, \& Twitter Facebook. (1970, January 1). Social media use increases depression and loneliness. Penn Today. https://penntoday.upenn.edu/news/social-media-use-increases-depression-and-loneliness.

28. Staff Correspondent Staff Correspondent. (2021, April 17). 16m become new poor in Bangladesh. The Daily Star. https://www.thedailystar.net/frontpage/news/16m-becomenew-poor-bangladesh-2079121. 
29. MJF: 4,249 women subjected to domestic violence during the lockdown. Dhaka Tribune. (2020, May 6). https://www.dhakatribune.com/bangladesh/2020/05/06/mjf-4-249women-endure-domestic-violence-amid-lockdown. 